\title{
Corela
}

Cognition, représentation, langage

HS-29 | 2019

Questions et exclamations au prisme de plusieurs approches linguistiques

\section{Les questions en anglais : une approche cognitive}

\section{Jérôme PUCKICA}

\section{(2) OpenEdition}

1 Journals

Édition électronique

URL : http://journals.openedition.org/corela/8905

DOI : 10.4000/corela.8905

ISSN : 1638-573X

Éditeur

Cercle linguistique du Centre et de l'Ouest - CerLICO

Référence électronique

Jérôme PUCKICA, «Les questions en anglais : une approche cognitive », Corela [En ligne], HS-29 |

2019, mis en ligne le 09 septembre 2019, consulté le 28 octobre 2019. URL : http://

journals.openedition.org/corela/8905; DOI : 10.4000/corela.8905

Ce document a été généré automatiquement le 28 octobre 2019

\section{(c) (i) (3) (2)}

Corela - cognition, représentation, langage est mis à disposition selon les termes de la licence Creative Commons Attribution - Pas d'Utilisation Commerciale - Partage dans les Mêmes Conditions 4.0 International. 


\title{
Les questions en anglais : une approche cognitive
}

\author{
Jérôme PUCKICA
}

\section{Préambule}

1 La linguistique cognitive (LC, angl. Cognitive Linguistics), telle que représentée par les publications de R.W. Langacker, G. Lakoff, L. Talmy, G. Fauconnier, J.R. Taylor, D. Geeraerts... n'est pas une théorie unifiée du langage, mais plutôt une façon d'aborder le langage et son étude qui repose sur l'adhésion à un certain nombre de principes fondamentaux: (a) le langage est d'abord un phénomène mental; (b) le savoir-faire linguistique ne constitue pas un système autonome au sein de l'«esprit/cerveau » humain, mais tout au contraire une "partie intégrante de la cognition» (Langacker 1987); (c) les hypothèses linguistiques doivent être compatibles avec les acquis et propositions des autres sciences cognitives, à commencer par la psychologie et la psycholinguistique; $(d)$ la grammaire est symbolique et non indépendante du sens : elle n'est pas mentalement représentée par des catégories et opérations purement formelles, mais par des réseaux de constructions et autres unités symboliques dotées de propriétés formelles et sémantiques-pragmatiques. Par suite, il n'existe qu'une différence de degré, et non de nature, entre le lexique et la grammaire : non pas une dichotomie, mais un continuum.

2 L'adoption de ces principes généraux et d'autres construits théoriques plus spécifiques n'exclut pas la possibilité de différences, voire même de divergences, entre les linguistes « cognitivistes» dans l'analyse de certains phénomènes. De fait, il n'existe pas de point de vue qui soit le point de vue de la LC sur les questions en anglais ou dans une autre langue. Dans les pages qui suivent, ce qui est proposé n'est donc qu'une approche cognitive des questions en anglais. Cette approche mobilise en particulier la Grammaire Cognitive (GC) développée par R.W. Langacker depuis le milieu des années 1980 (cf. Langacker 1987, 1990, 1991, 2008), dont les principes (a-d) ci-dessus résument très brièvement la philosophie. 


\section{Introduction}

Il existe plusieurs façons de définir ce qu'est une question en linguistique. En première approximation, on peut dire qu'une question est un énoncé au moyen duquel est accompli un certain acte de langage (illocutoire), qui est l'acte de questionner. Cet acte consiste typiquement en une demande d'information adressée par un locuteur L1 à un interlocuteur L2 dont il sollicite une réponse qui livre l'information demandée et comble ainsi le « déficit de connaissance » signalé par la question.

4 Selon le type de réponse appelé, on reconnaît traditionnellement au moins deux grandes classes de questions : (a) la question fermée ou totale, qui porte sur la totalité de l'énoncé qui l'exprime et appelle une réponse par « oui » ou «non », ex. (60) Did she ever talk about them $?^{1} ;(b)$ la question ouverte ou partielle, qui porte sur un constituant de l'énoncé qui l'exprime - typiquement le mot en wh en tête de proposition -, et sollicite une réponse qui identifie ou spécifie cet élément, ex. (89) What is his business?

Evidemment, toutefois, on ne pose pas uniquement des questions pour apprendre d'autrui ce que l'on ne sait pas. Dans une perspective cognitive ou cognitiviste, un acte de langage est d'abord un modèle ou scénario cognitif - « schème » ou « script cognitif » dans le vocabulaire des psychologues -, c'est-à-dire une structure de connaissances qui décrit une séquence type d'événements avec ses conditions de réalisation, ses participants, leurs attentes et leurs rôles, les règles ou normes socioculturelles associées, etc. ${ }^{2}$ Un tel scénario peut avoir des réalisations multiples et variées.

Le scénario de la question, considéré dans sa réalisation (proto)typique, peut être décrit comme suit : un locuteur L1 veut obtenir une information qu'il ne possède pas de son interlocuteur L2, pensant donc que ce dernier peut la lui donner. L'acte de question luimême, scène centrale du scénario, consiste pour L1 à demander à L2 de donner cette information. Le résultat immédiat en est que L2 se trouve en demeure de répondre ou "répartir " (reply, respond), même s'il n'est pas en mesure de donner la réponse attendue (answer), l'obligation étant plus ou moins forte selon la situation et les statuts respectifs des interlocuteurs. Idéalement, L2 fournit la réponse attendue à L1, auquel cas L1 possède lui aussi l'information au terme du scénario.

Dans une certaine mesure, les différents types de question peuvent être vus comme autant de variantes dans la réalisation de ce scénario. Certaines questions sont des offres (Would you like to come?), d'autres des requêtes indirectes (Could you please help me ?), d'autres encore des demandes de confirmation, notamment, nous le verrons, avec les question tags. Certaines questions n'attendent pas vraiment de réponse, comme les questions rhétoriques (Should we let those children die ?), qui sont souvent qualifiées de "fausses" questions (tant elles paraissent s'écarter du prototype) - elles tendent à fonctionner comme des assertions indirectes de polarité inverse de celle de la question. On peut aussi poser une question dont on connait la réponse pour vérifier si L2 la connaît aussi (ex. question d'examen ou de jeu), ou pour obtenir un aveu, etc. Il se peut aussi que le questionneur et le questionné soit la même personne, comme dans certains passages du texte au discours indirect libre, comme (2) When had that begun? What was the cause of it? Enfin, il va sans dire que L1 n'obtient pas toujours la réponse espérée : L2 peut ne pas connaître pas la réponse, ou ne pas vouloir la communiquer à L1, etc. Le texte en livre plusieurs exemples, comme (42) Well, I don't know exactly. 
Ci-dessous, nous examinerons d'abord la distinction entre les questions fermées et ouvertes et les structures propositionnelles interrogatives qui leur sont typiquement associées (\$1). Nous étudierons ensuite deux façons d'exprimer une question fermée autrement qu'avec une phrase interrogative fermée: le question tag et la question déclarative (\$2). Nous survolerons après cela le cas des "questions-répliques ", qui viennent en réplique à un premier énoncé, ex. (59) Afraid ?, avant d'étudier le cas des questions indirectes, ex. (30) Business, I suppose (\$4). Nous finirons par quelques remarques sur les subordonnées interrogatives (\$5), qu'il est parfois difficile de distinguer des constructions relatives dites libres ou sans antécédent, ex. (26) She thought she saw what was in his mind.

\section{Questions et phrases interrogatives}

9 La question compte parmi les actes de langage fondamentaux, au même titre que l'assertion, qui lui est complémentaire en tant qu'apport d'information, et que l'ordre, à qui elle s'apparente à certains égards (cf. \$1.3). ${ }^{3}$ De façon caractéristique, on trouve dans les langues des structures ou marques (prosodiques, morphologiques, syntaxiques) dont la fonction première est d'exprimer ces actes, notamment des types ou « modalités » de phrase : la phrase déclarative pour l'assertion, la phrase impérative pour l'ordre, et la phrase interrogative pour la question - ou plutôt les phrases interrogatives pour les questions. Toutefois, ici comme ailleurs dans la langue, il n'y a pas de correspondance biunivoque entre les structures et les fonctions: l'inversion sujet-auxiliaire caractéristique des phrases interrogatives en anglais, par exemple, a d'autres emplois dans cette langue (cf. \$1.4). De plus, une question n'est pas nécessairement marquée au sens strict du terme: sa valeur illocutoire peut être essentiellement affaire d'inférence pragmatique, comme dans certains cas de questions déclaratives (\$2.2) ou indirectes ( $\$ 4)$.

\subsection{Questions et phrases interrogatives fermées}

La question dite fermée ou polaire, ou yes-no question en anglais, appelle une réponse par yes ou no ou une réponse " équivalente ", comme l'illustre (23) Why, of course, George ( = 'Yes'). Elle ferme ainsi le champ des réponses possibles à une alternative polaire $(+/-) .{ }^{4} C^{\prime}$ est également une question dite totale plutôt que partielle, dans la mesure où elle porte sur la totalité de l'énoncé qui l'exprime. Elle peut prendre plusieurs formes, dont la plus canonique est la proposition interrogative fermée indépendante, soit la phrase interrogative fermée :

(60) 'Did she ever talk about them?'

(61) 'No, I don't think so.'

Dans une approche de la grammaire telle que la propose la GC, la phrase interrogative fermée de l'anglais peut être présentée comme un type de construction schématique ou "schéma constructionnel " doté de propriétés formelles et sémantiquespragmatiques. On le notera ici [ $\mathrm{AUX}_{\mathrm{F}}-\mathrm{S}-\mathrm{V}_{\mathrm{NF}}$ ?] ou encore [AUX $\mathrm{F}_{\mathrm{F}} \mathrm{S}$ - COMP ?] pour inclure $\left[\mathrm{Be}_{\mathrm{COP}}-\mathrm{S}-\mathrm{PC}_{\mathrm{S}}\right.$ ?]. ${ }^{5}$ Dans une telle approche, cependant, la connaissance des interrogatives fermées ne s'incarne pas dans une construction unique. Savoir produire et interpréter des phrases interrogatives, c'est avoir acquis et développé un vaste réseau interne d'unités symboliques qui présentent des degrés variables de complexité, 
de schématicité (abstraction) et d'ancrage cognitif (entrenchment), et qui sont liées entre elles par des relations diverses, notamment des relations catégorielles de schéma (type) à instance (cf. Langacker 1999, chap. 4).

Dans la GC, le savoir-faire linguistique émerge de la schématisation de l'expérience linguistique. Les locuteurs abstraient progressivement des représentations plus ou moins schématiques des structures de discours spécifiques auxquelles ils sont exposés de façon récurrente, le réseau se développant typiquement « de bas en haut » : ex. [Can $I \mathrm{~V}_{\mathrm{INF}}$ ?], [Would you $\mathrm{V}_{\mathrm{INF}}$ ?] --> [AUX $\mathrm{AOD}_{\mathrm{MOD}}-\mathrm{S}-\mathrm{V}_{\mathrm{INF}}$ ?] --> $\left[\mathrm{AUX}_{\mathrm{F}}-\mathrm{S}-\mathrm{V}_{\mathrm{NF}}\right.$ ?] ; des expressions ou tournures de phrases courantes peuvent également devenir des unités, ex. [Are you ready?], [Have you lost your mind?], [Do you think that...?], etc. (cf. Puckica 2015). C'est dans toute la richesse et la redondance du réseau qui peut ainsi se développer que s'incarne la connaissance des interrogatives fermées, non dans une unique construction maximalement abstraite dont toutes les autres pourraient être des instances. L'existence d'un «super-schéma » tel que $\left[\mathrm{AUX}_{\mathrm{F}}-\mathrm{S}-\mathrm{V}_{\mathrm{NF}}\right.$ ?] n'est d'ailleurs pas un postulat nécessaire : c'est une simple possibilité théorique à laquelle nous conférons surtout ici une utilité descriptive.

Ce point étant noté, nous pouvons examiner les propriétés de «la " construction interrogative fermée indépendante. D'un point de vue prosodique, elle se caractérise par une intonation finale montante, qui est plus généralement l'intonation par défaut des questions fermées en anglais. À elle seule, cette intonation peut d'ailleurs suffire à indiquer le caractère interrogatif d'un énoncé, ex. (50) ci-dessous. Cependant, les questions fermées avec une intonation finale descendante sont assez fréquentes dans le discours (oral) spontané. ${ }^{6}$ L'intonation descendante marque alors souvent une forme d'insistance, voire de menace (cf. Wells $2006: 45-46$ ), comme on peut le percevoir dans (49) et (51). George (L1) n'ordonne pas à Iris (L2) de répondre, mais ses questions se font plus pressantes ('dis-moi si P'). Incidemment, on remarquera que ces exemples, comme la plupart des interrogatives fermées du texte, contiennent des éléments «nonaffirmatifs » (ex. ever, much, any, at all) qui montrent que l'on ne peut pas toujours séparer clairement le contenu propositionnel (dictum, sémantique) de la valeur illocutoire (modus, pragmatique).

(49) 'Did Rosemary ever say she was aFRÀID of anybody?'

(50) 'AFRÁid?' Iris stared.

(51) 'What I'm trying to get at is, did Rosemary have any ènemies?'

D'un point de vue syntaxique, l'interrogative fermée indépendante présente une inversion sujet-auxiliaire (SA) qui implique l'emploi de $d o_{\mathrm{AUX}}$ en l'absence de tout autre auxiliaire, soit donc une structure du type $\left[\mathrm{AUX}_{\mathrm{F}}-\mathrm{S}-\mathrm{V}_{\mathrm{NF}} \ldots\right.$ ? ] ou $\left[\mathrm{AUX}_{\mathrm{F}}-\mathrm{S}-\right.$ COMP ... ?]. La question porte sur l'auxiliaire fini $A_{U X} X_{F}$ qui est placé en position initiale et qui constitue le noyau verbal de la proposition, de sorte que la question est totale : elle porte sur la réalisation du procès que décrit la proposition. ${ }^{7} \mathrm{~L} 1$ met en question la réalisation d'un procès et demande à $\mathrm{L} 2$ de trancher entre une réponse positive ou négative, soit d'exprimer sa position quant au «statut existentiel » du procès, i.e. la (non-)conformité du procès relativement à sa conception de la réalité (cf. Langacker 2008 : 470 sq., Langacker 2011). ${ }^{8}$

Une question fermée peut être plus ou moins « orientée » ou « dirigée » (biased) si une des deux réponses possibles paraît plus attendue que l'autre. Par défaut, l'interrogative fermée de polarité positive n'a pas d'orientation particulière et offre la façon la plus neutre de poser une question fermée. La présence de marques de doute ou 
d'incrédulité, particulièrement si elles sont accentuées, peut cependant suggérer une orientation négative: ex. Did she EVER/REALLY talk about them ? Par opposition, l'interrogative fermée de polarité négative a généralement une orientation négative :

(60) a. Did she ever talk about them? - Yes / No.

b. Didn't she ever talk about them? - (?)Yes / No / of course, she did!

Alors qu'il est possible de répondre à l'interrogative positive (60a) par un simple yes ou no, l'interrogative négative (60b) tend à appeler une réponse négative, tant et si bien qu'elle s'accommode assez mal d'un simple yes pour réponse. Typiquement, elle suggère que L1 a constaté un écart entre ce qui semble être et ce qu'il croyait être ou ce qu'il serait porté à croire. Ses croyances, passées ou présentes, ne correspondent pas à la réalité des faits telle qu'elle lui apparait. Dans (60b), par exemple, on peut imaginer que L1 croyait qu'elle avait parlé d'eux, mais que certains éléments l'ont amené à douter et qu'il s'attend maintenant à une réponse négative. Quirk et al. $(1985,808)$ proposent de même une distinction entre "old expectation" (positive) et "new expectation " (négative). Toutefois, l'orientation de la question est indissolublement liée à la sincérité du questionneur. Si elle exprime une question manifestement ironique ou rhétorique, $(60 \mathrm{~b})$ prend une orientation clairement positive, pouvant suggérer une réponse telle que of course, she did!

\subsection{Questions et phrases interrogatives ouvertes}

Tandis que les questions fermées peuvent être diversement exprimées, les questions dites ouvertes ou partielles ne le sont que par des propositions interrogatives " en wh", telles que (2) When had that begun? ou (89) What is his business? L'opposition terminologique de l'anglais entre les yes-no questions et les wH-questions reflète bien cette différence. La question en wh est une question partielle : typiquement, elle porte sur le mot en wh placé en tête de proposition et sollicite une réponse qui identifie ou spécifie le référent de ce mot. Prosodiquement, elle a par défaut une intonation finale descendante, contrairement à la question fermée, mais elle peut occasionnellement avoir une intonation montante "encourageante" (Wells 2006). ${ }^{9} \mathrm{Du}$ point de vue syntaxique, elle présente plusieurs variantes, notamment avec ou sans inversion sujetauxiliaire (SA) :

(2) When had that begun? $>\left[\right.$ Wh $-\mathrm{AUX}_{\mathrm{F}}-\mathrm{S}-\mathrm{V}_{\mathrm{NF}} \ldots$ ?]

(89) What is his business? $>\left[W h-V_{F} \ldots\right.$ ?]

L'inversion SA est une caractéristique générale des phrases interrogatives et c'est donc son absence qui est atypique. Elle est bloquée lorsque la question en wh porte sur le sujet (ex. What happened ? vs. *Did what happen ?) ou, mais presque uniquement avec be, sur l'attribut du sujet. Ce blocage ou préemption semble résulter de la priorité qui est donnée à un principe plus général : dans une phrase interrogative, la question porte sur l'élément placé en position initiale, l'élément « mis en avant » à tous les sens du terme, qui est soit $\mathrm{AUX}_{\mathrm{F}}$ (int. fermée), soit un mot en wh (int. ouverte). ${ }^{10}$ Ainsi, dans What is his business? (*Is what his business ?), l'initialisation du mot en wh semble l'« emporter » sur celle de is.

On peut noter deux cas particuliers. Premièrement, une préposition peut être placée en position initiale, ex. To whom am I talking? Cet agencement permet de maintenir la préposition devant son complément, donc dans sa position canonique, mais il contrevient au principe général d'initialisation du mot en wh sur lequel porte la 
question et, sauf autre blocage, c'est ce dernier qui tend à l'emporter dans la langue courante, ex. Who am I talking to? Deuxièmement, il existe des questions en wh «multiples », ex. Who did what to whom ? La question porte simultanément sur deux ou plusieurs mots en wh, mais seul un d'entre eux peut occuper la position initiale (puisqu'elle est unique) et les autres demeurent «in situ», dans ce qui est la position canonique des constituants correspondants dans une proposition déclarative.

Il existe une certaine variété catégorielle dans les mots en wh: pronom dans (89) What is his business?, déterminant dans (44) What sort of confidence do you mean?, "adverbe " dans (2) When had that begun? et (41) How intimate was she with them? Toutefois, ces mots sont sémantiquement homogènes en vertu du fait qu'ils contiennent tous le semi- ou sub-morphème interrogatif wh, y compris how [hav], dont who [hu:] est phonologiquement proche. ${ }^{11}$

21 Dans la GC, ce wh interrogatif n'est pas une marque de «déficit informationnel ». Il évoque plutôt la sélection d'une entité indéfinie (personne et sujet who, chose what, lieu where, moment when, etc.), parmi d'autres possibles, et plus spécifiquement, suivant Langacker (2001), le «chemin mental » qui mène du sujet conceptualisateur qui effectue la sélection à l'entité qu'il choisit (cf. \$5). Cette entité n'est autrement caractérisée, donc identifiable, que par son rôle dans le procès qu'exprime la proposition qui contient le mot en wh. Ainsi, dans (69) What did Sandra Farraday think about it?, le pronom what évoque la sélection d'une "chose » (plutôt que d'une personne ou d'un lieu, etc.) qui correspond au référent de l'objet du verbe think parmi un ensemble d'alternatives possibles. La question porte sur what, donc sur la sélection de cette chose. L'ensemble des alternatives possibles n'est pas explicitement délimité, mais s'il n'y avait aucune alternative, la question ne se poserait pas. C'est aussi ce qui fait de la question en wh une question «ouverte »: elle ne ferme pas le champ des réponses possibles par son seul contenu.

Les questions en wh sont parfois nommées variable questions en anglais, en ce sens que le mot en wh peut être conçu comme une variable dont la question demande de fixer la valeur. De ce point de vue, l'énoncé (69) demande quelle est la valeur de $x$ dans 'Sandra Farraday thought $x$ about it'. L'intérêt d'une telle formulation est de montrer que dans une question en wh, le procès lui-même est typiquement présupposé, i.e. présenté comme une situation déjà connue ou facilement "accessible " pour L2, soit encore comme de l'information ancienne ou d'arrière-plan plutôt que nouvelle. Ainsi, (69) présuppose 'Sandra Farraday thought something about it'. Cette présupposition est une distinction sémantique importante entre la question ouverte et la question fermée : (41) How intimate was she with them? présuppose 'She was intimate with them' et la question porte uniquement sur le degré d'intimité (how intimate ?). Par opposition, il n'y aurait pas de telle présupposition dans Was she intimate with them? Toutefois, comme dans d'autres cas, ce qui est considéré ou présenté par L1 comme déjà connu, acquis, évident etc. peut fort bien ne pas l'être pour L2. À la question (76) How did Rosemary and Farraday's wife get on ?, Iris réplique (77) I don't think they did, une réplique qui consiste précisément à nier le présupposé contenu dans la question.

\subsection{L'intonation des questions}

En anglais, les questions fermées tendent à recevoir une intonation finale montante tandis que les questions ouvertes tendent à recevoir une intonation finale descendante. 
Ces associations, nous l'avons noté, sont caractéristiques et non systématiques. De plus, l'opposition intonation montante vs descendante est une simplification qui ignore les degrés et la complexité des phénomènes intonatifs. Toutefois, la différence demeure et l'on peut se demander si elle a une raison d'être.

Il a souvent été proposé qu'il existerait dans les langues humaines une association entre (a) une fréquence fondamentale de la voix $\left(\mathrm{F}_{0}\right)$ comparativement basse ou descendante, qui est généralement caractéristique de l'assertion et de l'ordre, et ce qui est connu, certain, complet, définitif, ou encore des notions d'assurance, voire de domination ; et (b) une $\mathrm{F}_{0}$ haute ou ascendante, caractéristique de la question fermée, et ce qui est inconnu, incertain, incomplet, non-définitif, ou encore des notions de sollicitation, voire de soumission (cf. Ultan 1969, Ohala 1994, Vaissière 1995, Cruttenden 1997).

Bien que ces associations puissent paraitre relativement lâches, il est difficile de leur nier toute validité. Il existe, par exemple, des liens sémantiques évidents entre la certitude, l'assurance, la confiance (en soi), l'idée de s'imposer, d'ordonner et de dominer. On peut ainsi glisser de la notion de certitude à celle de domination et, parallèlement, de l'assertion à l'ordre. L'énoncé She's a writer est a priori une assertion si l'intonation est descendante, mais une question si l'intonation est montante. Un question tag d'intonation descendante (et de polarité inversée) n'est généralement pas une « vraie » question, mais plutôt une demande de confirmation, voire d'assentiment : l'intonation descendante y indique une plus grande assurance du locuteur. Dans une phrase interrogative fermée, l'intonation descendante tend à marquer une forme d'insistance, voire de menace : elle rend la question plus injonctive. Inversement, dans une interrogative ouverte, l'intonation montante est plus déférente ou encourageante : elle rend la question moins injonctive.

C'est peut-être également parce que le contenu de la question en wh est, pour l'essentiel, présupposé, donc considéré comme connu, acquis, etc., qu'elle a ordinairement une intonation finale descendante plutôt qu'ascendante, contrairement à la question fermée (cf. Lakoff \& Johnson 2003 : 137-8; Välimaa-Blum 2001: 4). Toutefois, puisque la question ouverte est bien une question, il pourrait sembler logique qu'elle ait, elle aussi, par défaut, une intonation montante. De fait, l'intonation des questions ouvertes dans les langues semble beaucoup plus variable que celle des questions fermées. Ultan (1969: 47), qui a examiné sur ce point 53 langues appartenant à plusieurs familles différentes, note une très large prédominance de contours intonatifs hauts ou montants dans les questions fermées (environ $72 \%$ à $89 \%$ ), mais indique que les questions ouvertes ont, à peu de choses près, des contours bas ou descendants dans la moitié des langues examinées (environ $52 \%$ ) et des contours hauts ou montants dans l'autre moitié (environ $48 \%$ ). La variabilité de l'intonation des questions ouvertes entre les langues pourrait s'expliquer par la concurrence de deux logiques: en tant que questions, elles sont compatibles avec un contour haut ou ascendant, mais dans la mesure où leur contenu est largement présupposé, elles sont compatibles avec un contour bas ou descendant. L'un ou l'autre des deux contours intonatifs pourrait ainsi devenir conventionnel, selon la logique adoptée.

Diverses hypothèses ont été avancées pour essayer d'expliquer les relations entre intonation et sens décrites plus haut. Lakoff \& Johnson (2003), par exemple, ont proposé qu'elles pourraient résulter de l'existence d'une métaphore conceptuelle qu'ils nomment « UNKNOWN IS UP; KNOWN Is DOWN », i.e. l'inconnu est en haut ou vers le 
haut, le connu est en bas ou vers le bas, métaphore qu'illustreraient aussi to raise a question / That's still up in the air / It's still up for grabs. / Let's bring it up for discussion / The matter is settled. ${ }^{12}$ Ces associations mentales auraient, selon les auteurs, un fondement expérientiel: il serait plus facile d'analyser, de comprendre et connaître ce qui se trouve " en bas ", sous nos yeux et devant nous, que ce qui se trouve " en haut ", audessus de nos têtes. Cette explication peut ne pas sembler très convaincante aux yeux du lecteur, mais la métaphore demeure intéressante, d'autant que parler d'intonation montante ou descendante, c'est parler de façon métaphorique.

Une autre hypothèse, avancée par Ohala $(1984,1994)$, est que les relations entre intonation et sens évoquées plus haut reposeraient sur un "code de fréquence " éthologique. Il existe une corrélation entre la masse corporelle et la fréquence fondamentale de la voix : les " gros » animaux tendent à produire des sons plus graves que les " petits » animaux (cf. Bowling et al. 2017). Pour cette raison, d'un point de vue éthologique, émettre des vocalisations graves pourrait être une façon de suggérer que l'on est gros ou grand, donc fort, sûr de soi, dominant. À l'inverse, les vocalisations aiguës suggéreraient que l'on est petit, faible, incertain, soumis. Par extension, cela pourrait expliquer les contours intonatifs caractéristiques des assertions et des ordres (certitude, domination) ainsi que des questions (incertitude, soumission), du moins des questions fermées. En d'autres termes, l'usage linguistique de l'intonation pourrait être une grammaticalisation de ce code de fréquence éthologique (cf. Nolan $2006: 439-40$ ). On notera toutefois qu'un principe de moindre effort pourrait également être impliqué dans la domination globale de contours descendants. On remarquera aussi l'ambiguité, voire le paradoxe de la position du questionneur, dans laquelle on peut voir tout aussi bien une soumission qu'une domination. Ignorant la réponse à sa question, le questionneur s'en remet aux connaissances du questionné et, dans une certaine mesure, se soumet à lui ; mais par sa question, il met aussi le questionné en demeure de répondre : il attend, voire exige de lui une réponse, donc s'impose également à lui. Ducrot (1983), cité par Kerbrat-Orecchioni (1991:28), décrit la question comme un «diktat» et parle du "pouvoir exorbitant que s'arroge le questionneur ». De fait, la question est un acte de langage qui est proche de l'ordre : l'ordre est une demande de faire, la question est une demande de dire et, c'est bien connu, «dire, c'est faire »! Certains pragmaticiens, comme Searle (1979: 13-14), ont d'ailleurs proposé de regrouper la question et l'ordre dans une catégorie supérieure d'actes "directifs » (directives).

\subsection{L'inversion sujet-auxiliaire}

29 L'inversion sujet-auxiliaire (inversion SA, ISA) est une des principales marques de la question en anglais. Elle est caractéristique des propositions interrogatives indépendantes, qu'elles soient fermées ou ouvertes, à l'exception des cas précédemment mentionnés (\$1.2). Soulignons que nous ne parlons ici d'inversion que dans le sens où l'ordre $\mathrm{AUX}_{\mathrm{F}}-\mathrm{S}$ que l'on peut observer dans les interrogatives indépendantes est inverse de l'ordre canonique $\mathrm{S}-\mathrm{AUX}_{\mathrm{F}}$ que l'on peut observer dans une proposition déclarative élémentaire: dans une approche cognitive de la grammaire, il n'existe pas de transformations, dérivations ou mouvements syntaxiques.

Du point de vue de la GC, il est envisageable que l'activation répétée de structures qui présentent une inversion SA mène progressivement à l'émergence d'un schéma 
constructionnel de haut niveau qui incarnerait ce qu'elles ont en commun et serait « la » construction ISA. Il pourrait s'agir d'une unité symbolique complexe dont la structure formelle spécifierait l'ordre $\left[\mathrm{AUX}_{\mathrm{F}}-\mathrm{S}-\mathrm{V}_{\mathrm{NF}}\right.$ ] ou $\left[\mathrm{AUX}_{\mathrm{F}}-\mathrm{S}-\mathrm{COMP}\right]$ sans lui associer de schéma intonatif montant, contrairement à $\left[\mathrm{AUX}_{\mathrm{F}}-\mathrm{S}-\mathrm{V}_{\mathrm{NF}}\right.$ ?]. Si l'on admet l'existence de cette construction - elle pourrait n'être qu'un artefact descriptif (cf. \$1.2) -, elle devrait aussi avoir un sens, un "pôle " sémantique. La difficulté est alors que l'inversion SA en anglais ne s'observe pas uniquement dans les phrases interrogatives: elle se rencontre également dans des exclamations (Boy, can she dance!), des souhaits ou vœux (May you find peace!), des hypothèses contrefactuelles (Had I known her intentions, ...), des phrases avec initialisation d'un adverbe négatif ou semi-négatif (Only then did I realise my mistake), ou encore positif-intensif (So absurd did it seem that...), et encore dans des propositions conjointes positives ou négatives (« rejoinders » : Neither/So was he).

Dès lors, il y a au moins deux hypothèses possibles. La première est qu'il y a un dénominateur sémantique commun à ces divers emplois, une sorte d'« invariant " sémantique. Goldberg (2006) et Langacker (2012) se rejoignent à peu près sur l'idée que l'inversion SA, étant donné qu'elle implique le placement de la tête de la proposition $\left(\mathrm{AUX}_{\mathrm{F}}\right)$ dans une position «non-canonique ", pourrait être l'indice formel du caractère sémantiquement-pragmatiquement marqué, «non-prototypique " (Goldberg 2006) de la proposition exprimée. L'ordre AUX-S(-V) indiquerait un écart par rapport à une sorte de "conception par défaut» (baseline conception, Langacker 2012), qui serait l'assertion positive non-emphatique qu'exprime la proposition déclarative élémentaire. D'autres grammairiens ont proposé de voir dans l'inversion SA de l'anglais une marque de "non-assertion » (Méry 1994) ou "le signe d'une relation prédicative dont la validation fait problème " (Garnier \& Guimier 1998: 128). Toutefois, ces propositions peinent à expliquer tous les cas d'inversion SA : par exemple, dans She was friendly and so was John, la structure conjointe so was John est bien assertée et l'on voit mal en quoi sa validation ferait problème.

Nous préférons, pour notre part, une autre hypothèse, qui est qu'il n'y a pas de dénominateur sémantique commun à tous les cas d'inversion SA en anglais. Le pôle sémantique de la construction ISA pourrait être une catégorie complexe organisée autour d'un prototype interrogatif: cet acte de langage fondamental qu'est la question servirait ainsi de prototype sémantique à la construction. De fait, si l'inversion SA a des emplois relativement variés, son emploi interrogatif paraît tout de même largement dominant. À ce prototype interrogatif se rattacheraient, directement ou indirectement, d'autres significations ou emplois (extensions ou élaborations). De cette façon, chaque membre de la catégorie pourrait être lié à au moins un autre, sans qu'il existe nécessairement de trait qui soit commun à tous. Par exemple, on peut raisonnablement lier la question à l'exclamation ainsi qu'à l'hypothèse, l'hypothèse au souhait, etc. Pour rendre compte des adjonctions positives comme (and) so was John, on peut alors postuler une extension de la construction ISA par analogie avec les adjonctions négatives comme (and) neither did John. Rappelons que la théorie du prototype a été précisément développée pour rendre compte du fait qu'il n'y a pas toujours de propriété commune à l'ensemble des membres d'une catégorie (cf. Taylor 2003). De plus, si l'on admet le continuum lexique-grammaire que postule la LC, alors il n'y a rien d'étonnant à ce que les unités ou constructions grammaticales soient polysémiques, puisque les unités lexicales le sont en très grande majorité. De ce point 
de vue, c'est en fait l'hypothèse d'une nécessaire unité sémantique derrière la diversité des emplois qui n'a pas de justification évidente (cf. Puckica 2008). ${ }^{13}$

\subsection{Le rôle de l'auxiliaire do}

Dans la majorité des phrases interrogatives fermées du texte (ex. 21, 43, 48, 50, 60), l'auxiliaire en position initiale est do, que l'inversion SA rend obligatoire en l'absence de tout autre auxiliaire. Du point de vue de la $\mathrm{GC}, d o_{\mathrm{AUX}}$ n'est pas un mot vide de sens dont la présence serait requise par des règles ou contraintes grammaticales purement formelles. C'est un verbe de schématicité maximale (Langacker 1991, 2012), une variante grammaticalisée du verbe lexical do dont le sens s'est affaibli au point de ne plus exprimer que la notion même de procès contenue dans tout autre verbe, soit la conceptualisation d'une relation qui se réalise ou existe dans le temps conçu. On peut remarquer que $d o_{\mathrm{AUX}}$ se construit aussi bien avec des verbes dynamiques comme talk qu'avec des verbes statiques comme seem, ce qui suggère qu'il n'est lui-même ni dynamique, ni statique : $d o_{A U X}$ transcende la distinction la plus fondamentale entre les types de procès et paraît bien à cet égard «maximalement schématique ». Par ailleurs, on peut faire l'hypothèse d'un glissement sémantique de $d o_{\text {LEX }}$ 'agir, faire, effectuer' (en concurrence partielle avec make, cf. Hirtle 1997) à do ${ }_{\text {AUX }}$ 'réalisation, accomplissement'.

Dans une structure $\left[\mathrm{do}_{\mathrm{AUX}}+\mathrm{V}_{\mathrm{LEX}}\right]$, do $\mathrm{ouX}_{\mathrm{AUX}}$ et $\mathrm{V}_{\mathrm{LEX}}$ désignent le même procès : $d \mathrm{o}_{\mathrm{AUX}}$ (fini) évoque la réalisation d'un procès schématique dont il permet le repérage grâce à sa flexion, tandis que $\mathrm{V}_{\mathrm{LEX}}$ (infinitif) en spécifie le type (cf. Langacker $\left.1991: 205\right)$. Do $\mathrm{AUX}_{\mathrm{AUX}}$ est le «squelette » de la construction, $\mathrm{V}_{\mathrm{LEX}}$ la «chair » qui le recouvre (Langacker 1990, $84) ; d o_{A U X}$ livre la forme, $V_{\text {LEX }}$ la matière notionnelle. $D o_{\text {AUX }} n$ 'apporte donc pas de contenu sémantique additionnel au GV, mais il permettrait, selon Langacker (2012: 35-6), d'exprimer séparément la réalisation du procès que spécifie $V_{\mathrm{LEX}}$ - son « noyau existentiel ». Les autres auxiliaires peuvent aussi jouer ce rôle, mais ils ne s'y réduisent pas : ils participent en plus de l'expression de distinctions «perspectivales " (aspect, voix) ou modales. Cela pourrait expliquer, d'une part, pourquoi $d o_{A U X}$ ne se combine pas avec d'autres auxiliaires et, d'autre part, pourquoi il apparaît en l'absence de tout autre auxiliaire dans les contextes où l'existence d'un procès est mise en cause ou « négociée » par les interlocuteurs, i.e. la question (Did he try ?), la négation (He didn't try) et l'assertion emphatique (He DID try).

À cet égard, il peut sembler cohérent que $d o_{\text {AUX }}$ ne s'emploie pas dans les propositions déclaratives positives non-emphatiques, où l'existence d'un procès est simplement posée. Cependant, cet emploi a existé par le passé et il existerait encore dans certains dialectes du sud-ouest de l'Angleterre, selon Palmer (1987:21) : il faut donc à nouveau reconnaître un certain degré de contingence dans la convention grammaticale. D'un point de vue historique, on notera également que la grammaticalisation de $d o_{\mathrm{AUX}}$ est relativement récente et que si les voies qu'elle a empruntées demeurent discutées, elle a, du moins a posteriori, toutes les allures d'un changement analogique qui a eu pour effet d'uniformiser le système verbal de l'anglais au regard de la construction des propositions, négatives, interrogatives et déclaratives emphatiques (cf. les « NICE properties $»)^{14}$

Enfin, on notera que $d o_{A U x} n$ 'exprime pas en lui-même la mise en cause d'un procès, de la même façon qu'il n'exprime pas en lui-même une assertion emphatique - le «emphatic 
DO » n'est qu'un emploi de $d o_{\text {AUX }}$ comme support d'un accent d'emphase (cf. Joly \& O'Kelly 1990 : 244) et l'on pourrait tout aussi bien parler de "negative DO » ou de " emphatic CAN ». Ainsi, dans des contextes de reprise, $d o_{A U X}$ peut très bien évoquer un procès dont la réalisation ou existence n'est pas (re)mise en cause, ex. I smiled and she did too (cf. Miller 2000). Dans cet emploi elliptique, commun à tous les auxiliaires, le fonctionnement de $d o_{\mathrm{AUX}}$ n'est pas différent de celui décrit plus haut: $d o_{\mathrm{AUX}}$ évoque la réalisation d'un procès schématique qui, pour être interprétable, doit être spécifié dans le contexte par un quelconque verbe ou GV.

\section{Question tags et questions déclaratives}

37 Les questions fermées sont canoniquement exprimées par des phrases interrogatives fermées, mais on les trouve également fréquemment exprimées par des question tags ou des questions déclaratives et il existe évidemment des différences entre ces constructions.

\subsection{Les question tags}

Le question tag (QT, angl. tag question) est un type de question fermée qui est adjoint à une proposition indépendante lui servant d'hôte ou d'« ancre ». Généralement, il vient à la suite de cette dernière et en est plus ou moins détaché, à la façon d'un circonstant de phrase. Il peut, sinon, être une incise dans la proposition hôte (ex. It's strange, isn't it, that we should meet again). On rencontre le QT essentiellement à l'oral, dans un registre plutôt familier, particulièrement dans des conversations privées (Kim \& Ann 2008). Le texte ne contient que des QT "variables", dont la forme varie en fonction de la proposition à laquelle ils sont adjoints, mais il en existe aussi des « invariables » (ex. right?, huh ?), qui sont ou s'apparentent à des interjections et sont comparables au fr. n'est-ce pas?, non?, pas vrai ?, hein?

Les QT variables présentent une certaine diversité de formes et de significations, selon le type de proposition auquel ils sont adjoints (ex. déclarative ou pas), sa polarité (inversée ou pas) et son intonation (montante ou descendante). Ils peuvent être envisagés comme formant une famille de constructions organisée autour d'un prototype, dont la figure 1 ci-dessous donne une représentation simplifiée et (94), un exemple concret :

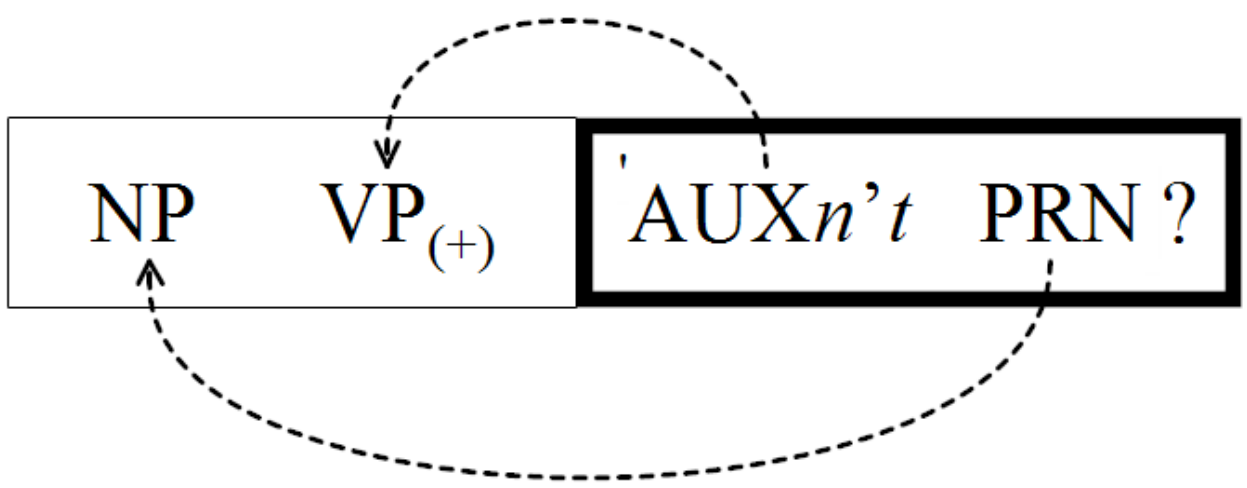

$40 \quad$ Fig. 1 


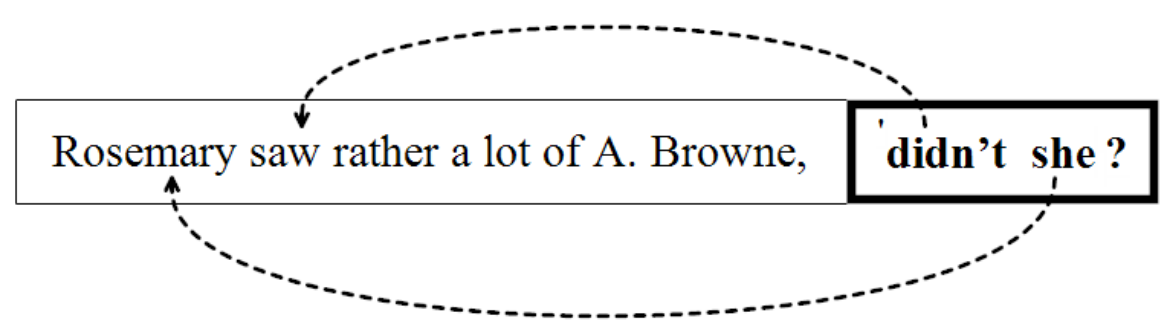

41 Le QT variable prototypique mêle à la fois des propriétés régulières et irrégulières ou idiosyncrasiques. Il offre ainsi une bonne illustration de l'intérêt de l'approche constructionnelle de la grammaire. ${ }^{15} \mathrm{D}$ 'un point de vue syntaxique, c'est une structure du type ['AUXn't $t_{\mathrm{HEAD}}+\mathrm{PRN}_{\mathrm{SUBJ}}$ ?] qui est ajoutée à la suite d'une proposition déclarative positive, le QT étant de polarité négative. Plus généralement, les QT de polarité inversée représentent l'immense majorité des cas, ceux de polarité constante étant comparativement rares. ${ }^{16}$ De même, on ne trouve qu'assez rarement des QT adjoints à des propositions autres que déclaratives.

Dans la construction prototypique, l'auxiliaire fini et le pronom sujet du QT reprennent ou s'accordent de façon régulière avec ceux de la déclarative hôte. La négation est elle aussi régulière et peut être attachée à l'auxiliaire ou, dans un registre plus soutenu, exprimée séparément en position finale, auquel cas l'accent tonique du groupe ne tombe pas sur l'auxiliaire (94a), mais sur not (94b). Par ailleurs, le QT ressemble à une interrogative fermée indépendante "réduite " à son auxiliaire principal et son sujet, mais ce n'est là qu'une apparence : le sujet du QT ne peut être qu'un pronom personnel, l'explétif there, ou exceptionnellement l'indéfini one, mais pour ainsi dire rien d'autre, donc pas un nom propre ou un GN simple (94c). De plus, la structure ne peut pas être "développée ", un verbe lexical ou un quelconque adverbe ne pouvant pas être ajouté (94d) :

(94) a. Rosemary saw rather a lot of Anthony Browne, DiDn't she?

b. Rosemary saw rather a lot of A. Browne, did she Not?

c. *She saw rather a lot of A. Browne, didn't \{Rosemary / the girl\}?

d. *Rosemary saw rather a lot of A. Browne, didn't she \{see him / often / a lot\}?

Les autres exemples du texte sont du même type, si ce n'est pour le caractère partiellement elliptique de certains - ellipse du sujet (98), ou du sujet et de l'auxiliaire de la déclarative $(81,91)$. L'exemple (110) discuté plus bas s'écarte davantage du prototype mais présente également une proposition déclarative positive suivie d'un QT négatif.

D'un point de vue sémantique, l'interprétation du QT de polarité inversée dépend fortement de son intonation, montante ou descendante. Le QT d'intonation montante marque l'incertitude (" UNKNOWN IS UP ») et signale typiquement une " vraie » question. Faisant suite à une assertion, il suggère un revirement de L1, qui soudainement doute et demande à L2 son avis. Par opposition, le QT d'intonation descendante indique une plus grande assurance de L1 (« KNOWN IS Down ») : c'est une demande de confirmation, voire une simple invitation à donner un signe d'acquiescement ou d'adhésion. ${ }^{17}$ Le texte-corpus étant un document écrit, on peut parfois douter de l'intonation qu'il convient de donner à un QT, mais il semble toutefois possible de distinguer les exemples suivants :

(94) 'Rosemary saw rather a lot of Anthony Browne, DíDn’t she?

'Yes - yes, she did.' 
(84) ‘Knocked about a good deal, $\underline{\text { HÀsn't he? }}$ Must have had an interesting life. Does

he

ever talk to you about it?'

'Not much. He's travelled a lot, of course.'

Dans (94), on peut penser que le QT a une intonation montante et exprime une vraie question qui appelle une réponse de L2 : la réplique de Iris est une triple confirmation. Inversement, dans (84), le QT semble plutôt avoir une intonation descendante : George continue de parler et ne cède donc pas la parole à Iris, ce qui suggère que sa « question "n'en est pas vraiment une. Le QT est davantage une façon de vérifier si Iris est d'accord avec l'assertion qui précède, voire simplement une façon de prendre Iris à témoin et de l'inviter à suivre son propos.

Le QT étant une sorte de circonstant de phrase, son interprétation est également indissociable de la proposition à laquelle il est adjoint et il diffère nécessairement en cela d'une simple interrogative fermée indépendante :

(91) a. It's something to do with armament firms, isn't it? (= 91 avec ajout de it's)

b. Is it something to do with armament firms?

L'interrogative fermée (b) exprime un seul acte de langage. C'est, par défaut, une question non-orientée portant sur la réalité d'une situation: L1 « ne sait pas » et demande à L2 de trancher entre une réponse positive ou négative. Par opposition, (a) est une structure en deux temps et en deux actes (de langage), avec d'abord une affirmation, puis une question dont l'interprétation varie selon l'intonation du QT. Même si ce QT exprime une vraie question, il demeure que ce qui est mis en question dans (b) commence par être affirmé dans (a) : plutôt que de demander une information, L1 commence par apporter une information puis en vérifie la validité auprès de L2. Pour cette raison, la phrase avec un QT peut offrir un mode de questionnement moins abrupt, voire plus subtil, que la simple interrogative fermée. Cela pourrait en partie expliquer pourquoi les QT sont particulièrement fréquents dans les conversations privées, où la volonté d'obtenir des renseignements de L2 peut être en conflit avec celle de ne pas sembler exiger de lui une réponse.

Enfin, on considérera brièvement l'exemple de la dernière ligne du texte :

(110) Such a gay party it had been, or hadn't it?

Le QT de cet exemple présente un certain nombre de particularités: (a) il semble exprimer un véritable doute quant à la réalité de la proposition précédente, mais son intonation est a priori descendante, peut-être en partie parce que c'est une question que Iris s'adresse à elle-même, dans ses pensées (discours indirect libre), et qui ne requiert donc pas de marque explicite d'appel à autrui ; (b) c'est un QT de polarité négative qui est ajouté à la suite d'une proposition déclarative positive, mais celle-ci n'est pas une simple assertion: elle a un sens exclamatif, auquel contribue l'antéposition du GN attribut such a GAY party. Il s'agit toutefois bien d'une déclarative, et non d'une exclamative, comme What a gay party it had been!; (c) le QT est précédé de la conjonction-disjonction or, qui marque une séparation entre les deux parties de la phrase et introduit une notion d'alternative à la proposition qui précède. Sa présence s'explique peut-être par le caractère exclamatif de la première proposition: une exclamation est rarement suivie d'un QT qui la met véritablement en question, l'enthousiasme («oui ! ») et le doute («non?») pouvant difficilement être exprimés dans un même mouvement de pensée. Or pourrait servir à marquer cette opposition et la transition d'un premier état d'esprit à un autre, fort différent. 


\subsection{Les questions déclaratives} orienter particulièrement la réponse de $\mathrm{L} 2$. Avec la $\mathrm{QD}$ positive (b), L1 demande à L2 s'il confirme ou prend en charge l'assertion positive. Par l'emploi de (b) dans le texte, George (L1) suggère à Iris (L2) qu'il est porté à croire en la validité de l'affirmation correspondante (e). Le lecteur, cependant, comprend que George demande ici la confirmation d'une affirmation à laquelle il feint de croire et qu'en son for intérieur il pense que A. Browne (he) pourrait avoir été l'amant de Rosemary. Si cette duperie est possible, c'est parce que la QD a un sens : c'est une construction grammaticale dotée de propriétés non seulement formelles, mais aussi sémantiques-pragmatiques, tout comme la construction interrogative fermée indépendante. 
trouve aux lignes 52-3 du texte une autre QD, mais de polarité négative (a), que l'on peut à nouveau comparer à l'interrogative fermée correspondante (b) :

(52) a. There wasn't anyone - that you knew of - who - who might have had it in for her?

b. Wasn't there anyone - that you knew of - who - who might have had it in?

A l'oral, le sens interrogatif de (52a) peut s'inférer de son intonation, que l'on imagine montante sur la fin, mais aussi de l'incise that you knew of (par laquelle L1 fait appel aux connaissances de L2) et du modal might (indiquant l'incertitude de L1), voire peut-être aussi des pauses que marquent les tirets et de la répétition de who, qui suggèrent toutes deux l'hésitation. Il ne s'agit pas là de marqueurs de la question au sens strict, mais néanmoins d'indices sur la base desquels un énoncé déclaratif, même d'intonation descendante, peut être interprété comme une question (cf. \$4). Quant à la différence entre (52a) et (52b), elle est plus fine qu'entre (97a) et (97b) puisque la QD et l'interrogative fermée négatives ont toutes deux une orientation typiquement négative. Il y a cependant une différence de degré : par défaut, la QD est plus proche de l'assertion que l'interrogative. Avec la QD négative, c'est l'assertion de la nonréalisation du procès qui est mise en question; avec l'interronégative, c'est la nonréalisation du procès elle-même : $\mathrm{L} 1$ adopte une position plus distante par rapport à l'assertion et son incertitude parait plus grande. Toutefois, on notera à nouveau la complexité sémantique des questions fermées négatives. En demandant à Iris de confirmer la non-réalité d'une situation (there wasn't anyone?), George lui révèle inévitablement qu'il a envisagé la possibilité de sa réalité, d'où la suite du texte: (55) Sounds silly, I know. Melodramatic, but I just wondered.

\section{Les questions-répliques}

La «question-réplique» $(\mathrm{QR})$, souvent nommée echo question en anglais, est une question ouverte ou fermée dont la forme tend à être plus ou moins tronquée et qui vient en réplique à un premier énoncé ou « stimulus » (Huddleston $2002: 855$ ). Celui-ci est souvent lui-même une question, comme c'est le cas dans tous les exemples du texte :

(68) George said: 'What did Sandra Farraday think about it?'

(69) 'About what?'

Nous empruntons l'expression question-réplique à Grésillon (1981:68), pour qui elle « regroupe tous les cas où une question revient (pour faire répéter ou préciser) sur une assertion ou question antérieure. » En anglais, on parle aussi de repair questions, i.e. des questions qui visent à réparer une partie « défectueuse » de l'échange conversationnel. La QR n'est donc pas, par exemple, une question retournée (ex. A : Do you know what time it is ? - B : Do you?), qui constitue un refus de répondre, soit de coopérer, et qui est donc potentiellement dommageable au bon déroulement de la conversation.

En schématisant, on peut reconnaître deux grands types de QR : (a) la demande de clarification ou précision, qui porte plutôt sur le contenu d'un premier énoncé ; et (b) la demande de répétition, qui porte plutôt sur sa forme, soit dans une certaine mesure une question métalinguistique. À une exception près, les $Q R$ du texte sont toutes du premier type, comme (69) ci-dessus, où Iris demande à George de clarifier la référence du it anaphorique contenu dans son énoncé; ou encore (23) ci-dessous, dans laquelle elle demande à George de préciser le référent de l'argument implicite de talk : 
(21) 'Look here, Iris, did Rosemary ever talk to you much?'

(22) Iris stared at him.

(23) 'Why, of course, George. At least - well, about what?' tant qu'acte de langage indirect, soit une question implicite, exprimée par un énoncé qui n'est pas formellement interrogatif; (b) la question rapportée au discours indirect au moyen d'une proposition subordonnée interrogative. Nous ne parlerons ici de question indirecte que dans le sens (a). Les deux sens, toutefois, se recoupent en partie, puisqu'une subordonnée interrogative peut participer de l'expression d'une question indirecte : ex. I wonder how she did it peut être une façon détournée de demander à L2 How did she do it?; et I wonder how you did it se comprendrait presque inévitablement comme une question.

Laestion indirecte peut être préférée à la question directe par politesse ou tact, car elle n'a pas la force injonctive de la question directe, qui met L2 en demeure de répondre. Secondairement, il peut aussi s'agir d'une stratégie de dissimulation, par laquelle L1 essaie d'obtenir des renseignements de L2 sans trop dévoiler sa démarche.

(30) '[...] I thought she might have said something.'

He looked at her inquiringly - rather like a hopeful dog.

She didn't want George to be hurt. And anyway Rosemary never had said anything.

She shook her head.

George sighed. He said heavily: 'Oh, well, it doesn't matter.' 

continuum assertion-question précédemment évoqué. C'est à la fois une assertion (directe) et une question (indirecte). De façon caractéristique, (30) contient des marques d'incertitude de L1 (thought, might) quant à la réalité d'une situation qui, de surcroît, est a priori mieux connue de L2, puisque (30) signifie bien 'I thought she might have said something to you'. La suite immédiate du texte confirme que (30) est bien conçu comme une question: George adresse à Iris un regard "interrogateur ", qui est comparé à celui du chien fidèle qui attend patiemment un geste de son maitre - une image du caractère soumis du questionneur qui rappelle le paradoxe de sa position (cf. §1.3). Cet énoncé illustre également l'importance d'éléments paralinguistiques dans la communication: gestes, expressions faciales, regard, ton de la voix, etc. Autant d'éléments qui peuvent d'ailleurs accompagner la parole ou s'y substituer: She shook her head est une façon de dire que Iris a répondu "non ", confirmant qu'elle a bien perçu la valeur interrogative de (30).

Les autres questions indirectes du texte sont similaires à (30) :

(73) '[...] Sort of woman who might resent his having a friendship with another woman.'

'Perhaps.'

Comme (30), (73) a une structure déclarative (elliptique : She is the sort of woman...) et l'intonation finale descendante d'une assertion. À nouveau, George (L1) y exprime son incertitude (might) concernant une situation mieux connue de Iris (L2), puisque cette dernière connait personnellement la femme en question, alors que George ne semble la connaître que de vue et par ouï-dire (cf. ligne précédente : Looks cold as ice. They say she's crazy about Farraday). A la suite de (73), on pourrait facilement ajouter « don't you think? » et la réplique de Iris confirme que l'énoncé est bien perçu comme une question.

On peut également mentionner, à propos des raisons qui pourraient expliquer pourquoi A. Browne a beaucoup voyagé : (87) 'Business, I suppose.' / (88) 'I suppose so.' Là encore, dans un énoncé elliptique, mais tout de même plus déclaratif qu'interrogatif et d'intonation finale descendante, George exprime une incertitude (I suppose) quant à une situation que Iris connaît mieux que lui, puisqu'elle fréquente A. Browne alors que George ne le connait pas personnellement.

On peut s'interroger quant aux bases du fonctionnement des actes de langage indirects et, en particulier, de la question indirecte, i.e. se demander comment un énoncé qui n'est pas formellement interrogatif peut néanmoins être conçu et/ou interprété comme une question. En fait, comme nous l'avons vu, les exemples du texte présentent tous des indices de leur valeur illocutoire, ces mêmes indices que l'on a pu relever dans des questions déclaratives (\$2.2) : certains expriment le doute ou l'incertitude de L1 (ex. might, I suppose), d'autres renvoient à L2 et/ou ses connaissances (you know).

71 Une hypothèse générale serait qu'il existe une sorte de déontologie de la communication qui implique une entraide ou coopération des interlocuteurs (cf. Grice 1975) et, en particulier, un partage de l'information qu'ils possèdent - «si tu vois que je ne sais pas, que je doute ou que je m'interroge, et que tu connais la réponse, alors tu dois me la donner "; «si tu sais que je me trompe, tu dois me le dire »-, étant bien évidemment entendu que les locuteurs ne font pas toujours preuve de déontologie ou d'altruisme et que ce partage de l'information n'est pas toujours possible, ni même souhaitable. 
72 Dans le cadre de la LC, une hypothèse complémentaire est que les actes de langage indirects seraient des «métonymies illocutoires» (Panther \& Thornburg 1998, 2007). Du point de vue de la LC, les actes de langage sont des scénarios cognitifs complexes reposant sur un vaste arrière-plan de connaissances socioculturelles (cf. introduction). La théorie des métonymies illocutoires, résumée en quelques mots, est qu'une partie d'un tel scénario, si elle est suffisamment centrale, peut, par métonymie, servir à évoquer le scénario dans son ensemble, ou du moins sa scène cruciale («core »), de la même façon que l'on peut faire référence à une entité en évoquant une de ses parties ou propriétés saillantes (ex. un rouge-gorge, une toile de maittre, un coureur de jupons, etc.). Dans une réalisation typique du scénario de la question, L1 cherche à obtenir une information qu'il ne possède pas de L2 et suppose donc que L2 possède ou pourrait posséder cette information. La métonymie illocutoire de la question indirecte consisterait, peut-on penser, à évoquer cet aspect du scénario de la question pour faire référence à sa scène centrale, i.e. pour demander l'information. Cette analyse est cohérente avec la présence fréquente, dans les questions indirectes (et déclaratives), des indices mentionnés plus haut (I suppose, you know, etc.). ${ }^{19}$

\section{Les subordonnées interrogatives}

73 Les propositions subordonnées interrogatives peuvent, comme les indépendantes du même nom, être réparties en interrogatives fermées, introduites par if ou whether, incluant les alternatives (ex. 24-5), et interrogatives ouvertes, introduites par un mot en wh (ex. 14, 37). ${ }^{20}$

Cependant, les interrogatives subordonnées diffèrent considérablement des interrogatives indépendantes. Formellement, elles ne présentent normalement pas d'inversion SA en anglais standard, ni d'intonation montante pour l'interrogative fermée, ni de point d'interrogation à l'écrit. D'un point de vue sémantiquepragmatique, elles ne sont pas, à proprement parler, des questions, de même que les subordonnées déclaratives (en that) ne sont pas des assertions : elles n'en ont pas la force illocutoire. Une subordonnée interrogative ne pose pas une question, mais permet d'en évoquer une, implicitement ou explicitement, soit éventuellement d'en rapporter une (au discours indirect). Elle apparaît souvent dans un contexte qui évoque lui-même le questionnement, l'incertitude, le doute, la curiosité, etc., sa présence étant alors liée au sens « interrogatif » du mot dont elle dépend - verbe (ask, wonder, doubt...), adjectif (unsure, uncertain, doubtful...) ou nom (question, issue...) - et/ou à la polarité de la proposition matrice (I don't know if... / I'm not sure whether...) ou encore à son type ou sa modalité (Do you know if... / Soon he would know whether she had lied). Toutefois, les subordonnées interrogatives ne sont pas limitées à de tels contextes :

(37) a. he asked her who Rosemary's friends had been (監 37)

a'. he asked her, 'who were Rosemary's friends?'

a". he asked her \{something / *someone\}

b. he told her who Rosemary's friends had been

b'?*he told her, 'who were Rosemary's friends?'

b". he told her \{something / *someone\}

c. he knew who Rosemary's friends had been

c'. *he knew, 'who were Rosemary's friends?'

c". he knew \{something / someone\} ${ }^{21}$ 
Dans (a), la subordonnée en who est une question rapportée et il est possible de lui faire correspondre une question directe $\left(a^{\prime}\right)$. Toutefois, ces propriétés dépendent du verbe (ask) qu'elle complémente: tell ( $\left.b^{\prime}\right)$ et encore moins know ( $\left.c^{\prime}\right)$ ne permettent cette correspondance. De même, (a) implique he asked her a question, mais (b) n'implique pas he told her a question et (c) n'implique pas he knew a question. Un substitut plus adéquat dans (b-c) serait éventuellement et paradoxalement the answer (to the question who R.'s friends had been). Dans les trois cas (a-c), cependant, la subordonnée en who est bien une interrogative et non une relative libre (" sans antécédent ", " nominale ", " headless ", " fused », etc.). Il existe divers tests permettant de les distinguer (cf. Leonarduzzi 2004). Notons simplement qu'une subordonnée interrogative a un contenu propositionnel et que l'on peut donc éventuellement lui substituer un GN à référent inanimé, mais pas un GN à référent humain. En revanche, une relative libre en who désigne une ou plusieurs personne(s), spécifique(s) ou pas. Elle est donc éventuellement remplaçable par un GN à référent humain, mais pas à référent inanimé : ex. You cannot marry just $\{w h o(m)(e v e r)$ you want / anyone / *anything\}. Or, dans (a-c), la subordonnée en who peut être remplacée par something, mais pas par someone (a"-c").

Des exemples tels que (37b-c) peuvent sembler problématiques si l'on estime qu'un mot interrogatif en wh indique toujours un « déficit informationnel » ou « vide référentiel ». Suivant Girard (2001), il ne pourrait donc s'agir que de "fausses interrogatives », qui représenteraient une catégorie distincte à la fois de l'interrogative (véritable) et de la relative. Langacker (2001: 148) propose une analyse du submorphème wh qui offre une solution intéressante à ce problème. Cette analyse est fondée sur une analogie sémantique avec le nom choice, dont trois sens sont reconnus, associés chacun à une représentation diagrammatique distincte: (a) She only needed a few seconds to make her choice $_{1}$. / (b) Her choice ${ }_{2}$ turned out to be a competent manager. / (c) Her choice ${ }_{3}$ is still a secret.

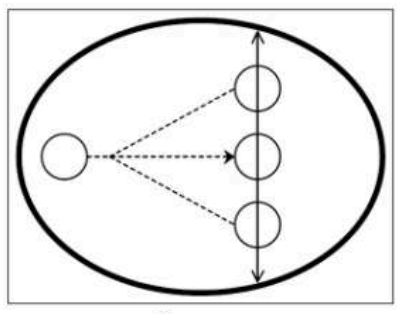

choice $_{1}$

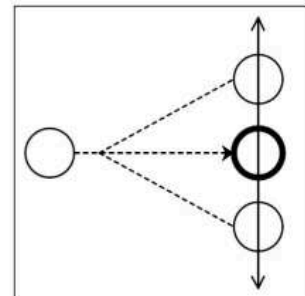

choice $_{2}$

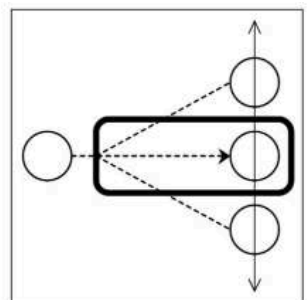

choice $_{3}$

77 En simplifiant quelque peu, choice évoque l'acte de choisir dans son ensemble, soit une conception réifiée (nominalisation) du procès que dénote le verbe choose. Choice désigne l'entité choisie parmi celles possibles, correspondant à ce qui serait l'objet de choose dans un emploi transitif simple. Cette entité existe indépendamment du choix et peut être une personne concrète, comme dans (b), où her choice ${ }_{2}$ est remplaçable par (s)he. Enfin, choice ${ }_{3}$ désigne la trajectoire mentale abstraite ou "chemin mental » (mental path) qui va de la personne qui choisit à l'objet de son choix. Ce chemin mental n'a pas, quant à lui, d'existence indépendante du choix et il est nécessairement abstrait : c'est une chose abstraite, comme dans (c), où her choice ${ }_{3}$ peut être remplacé par it, mais pas par (s)he.

Suivant Langacker (2001), la distinction sémantique entre choice ${ }_{2}$ et choice ${ }_{3}$ serait analogue à celle entre la variante relative libre et la variante interrogative de wh, si ce n'est qu'avec wh l'entité sélectionnée est en plus repérée par rapport à un procès $P$, i.e. 
caractérisée par son rôle dans le procès que dénote la structure qui contient le mot en wh. C'est ce que vise à représenter les diagrammes ci-dessous adaptés de Langacker (id.). Cette analyse de wh permet notamment de rendre compte des différences notées plus haut entre les possibilités de substitution des subordonnées interrogatives en who et celles des relatives libres en who.

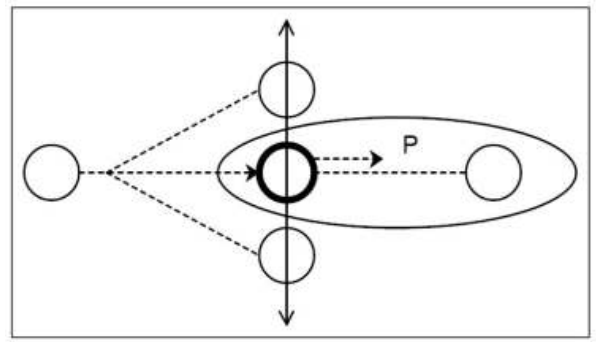

wh- relatif libre

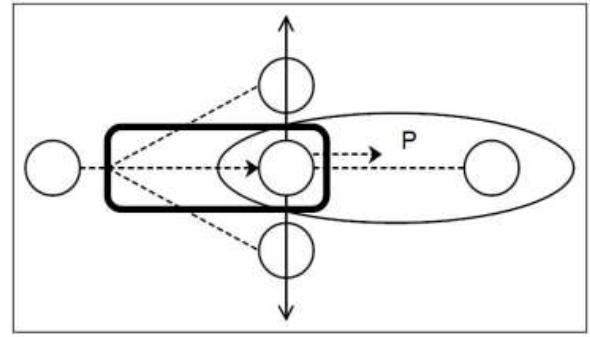

$w h$ - interrogatif dans le sens relatif, 'the thing that was in his mind', 'that which was in his mind' ; (b) dans le sens interrogatif, le "chemin mental » qui mène à cette chose, sa sélection, dont on peut également considérer la glose 'the answer to the question (as to) what was in his mind'. Dans (26), cette structure complémente une forme du verbe see qui a plutôt le sens de 'comprendre, se rendre compte' (comme dans le fr. je vois ce que vous voulez dire). See y est donc davantage un verbe psychologique qu'un verbe de perception, ce qui peut suggérer un what interrogatif. Cependant, ce sens figuré est dérivé du sens premier de perception visuelle, dans lequel l'objet de see peut être abstrait (ex. I see the light > I see hope/joy around me >I see the problem). Sans plus de contexte, une lecture relative de she saw what was in his mind ne semblerait donc pas entièrement exclue. De fait, c'est le contexte plus large qui tend à lever l'ambiguïté et à imposer une lecture interrogative. Iris s'étonne des questions que lui pose George ; elle trouve son comportement curieux (cf. (20) 'queer'), donc s'interroge. L'emploi de thought (She thought she saw...) indique également son incertitude et renforce la lecture interrogative. Enfin, la phrase qui suit - He must have got wind... - peut se comprendre comme une réponse, elle-même incertaine (must), à la question qu'évoque implicitement what was in his mind dans son interprétation interrogative. Ce dernier exemple illustre assez bien la difficulté qu'il peut y avoir à distinguer une subordonnée interrogative en wh d'une construction relative libre. Il n'est pas étonnant que ces structures fassent encore l'objet d'autant de désaccords entre grammairiens.

\section{Conclusion}

Cette étude des questions dans un extrait du roman Sparkling Cyanide (1945) de A. Christie nous aura permis d'entrevoir toute la complexité et la richesse des questions et plus généralement, à travers elles, des actes de langage. 
La question est un scénario cognitif aux réalisations multiples et variées. Il existe ainsi une remarquable diversité de formes et de types de questions : directes ou indirectes, fermées ou ouvertes, de forme interrogative ou déclarative, d'intonation montante ou descendante, "vraies » ou "fausses ", adressées à autrui ou à soi-même, etc. De plus, il existe des liens entre la question, l'assertion et l'ordre qui font apparaître les actes de langage fondamentaux comme des catégories radiales aux contours plus ou moins flous. On peut, en particulier, reconnaître un continuum question-assertion qui va de la demande d'information la plus neutre telle que peut l'exprimer une proposition interrogative positive à l'apport d'information que peut exprimer une proposition déclarative élémentaire. Cependant, la question est également proche de l'ordre par son caractère " directif », et l'ordre présente à son tour des similitudes avec l'assertion, notamment d'un point de vue intonatif. En outre, certains énoncés sont pragmatiquement hybrides, pouvant notamment être à la fois des assertions (directes) et des questions (indirectes), ou des questions (directes) et des requêtes (indirectes), etc.

Enfin, cette étude aura tenté d'illustrer la pertinence d'une approche cognitive ou cognitiviste du langage qui admet l'existence de catégories grammaticales complexes organisées autour de prototypes, et dans laquelle les connaissances grammaticales s'incarnent, tout comme les connaissances lexicales, dans de vastes réseaux de constructions et autres unités symboliques. Une telle approche du langage n'ignore pas la contingence et les aléas de l'usage, mais la grammaire, loin d'y être considérée comme un système purement formel et indépendant du sens, y est tout au contraire envisagée comme étant fondamentalement signifiante et motivée.

\section{BIBLIOGRAPHIE}

Bowling, D.L., Garcia, M., Dunn, J.C., Ruprecht, R., Stewart, S. Frommolt, K.-H. \& Fitch, W.T. (2017). Body size and vocalization in primates and carnivores. Scientific Reports, 7, 41070.

Chen, R. (2013). Subject auxiliary inversion and linguistic generalization: Evidence for functional/ cognitive motivation in language. Cognitive Linguistics 24, 1, 1-32.

Croft, W. \& Cruse, A. (2004). Cognitive Linguistics. Cambridge: Cambridge University Press.

Cruttenden, A. (1997). Intonation. 2nd ed. Cambridge: Cambridge University Press.

Ducrot, O. (1983). La valeur argumentative de la phrase interrogative. In Bange, P. et al. (eds)

Logique, argumentation, conversation. Actes du Colloque de Pragmatique, Fribourg, 1981. Berne /

Francfort : Peter Lang, 79-110.

Filppula, M. \& Klemola, J. (2007). Celtic influence on English: a re-evaluation. In Cloutier, R.A., A.M. Hamilton-Brehm \& W.A. Kretzschmar, Jr. (eds.) Studies in the History of the English Language V. Berlin / New York: W. de Gruyter, 207-225.

Garnier, G. \& Guimier, C. (1998). L'épreuve de linguistique au CAPES et à l'agrégation d'anglais. Paris : Nathan Université. 
Girard, G. (2001). Les fausses interrogatives. In L'interrogation. Actes du colloque du 17/03/2001, Univ. Paris III. SESYLIA.

URL : https://alaesfrance.files.wordpress.com/2015/04/girard.pdf

Goldberg, A. (2006). Constructions at Work. Oxford: Oxford University Press.

Goddard, C. \& Wierzbicka, A. (2004). Cultural scripts: What are they and what are they good for? Intercultural Pragmatics 1-2, 153-166

Grésillon, A. (1981). Interrogation et interlocution. DRLAV 25, 61-75.

Grice, H.P. (1975). Logic and conversation. In Cole, P. \& J.L. Morgan (eds.) Syntax and Semantics, vol. 3: Speech Acts. New York: Academic Press, 41-58.

Hirtle, W. (1997). DO auxiliary: A meaningful support and operator. Lingua 100(1), 111-149.

Huddleston, R. (2002). Clause type and illocutionary force. In Huddleston, R. \& G.K. Pullum (eds.) The Cambridge Grammar of the English Language. Cambridge: Cambridge University Press, 851-945.

Joly, A. \& O'Kelly, D. (1990). Grammaire systématique de l'anglais. Paris: Nathan Université.

Kerbrat-Orecchioni, C. (1991). Introduction. In Kerbrat-Orecchioni, C. (ed.) La question. Lyon : Presses Universitaires de Lyon, 5-37.

Kim, J.-B. \& Ann, J.-Y. (2008). English Tag Questions: Corpus findings and theoretical implications. Paper presented at the 2008 Northwest Linguistics Conference, May 3-4, University of Washington, Seattle.

URL: http://web.khu.ac.kr/ jongbok/research/final-papers/eng-tag-jn-sub-2.pdf

Lakoff, G. \& Johnson, M. (2003). Metaphors We Live By. 1st ed. 1980. Chicago / London: The University of Chicago Press.

Langacker, R.W. (1987). Foundations of Cognitive Grammar, vol. I: Theoretical Prerequisites. Stanford, Ca.: Stanford University Press.

Langacker, R.W. (1990). Concept, Image, and Symbol: The Cognitive Basis of Grammar. Berlin / New York: Mouton de Gruyter.

Langacker, R.W. (1991). Foundations of Cognitive Grammar, vol. II: Descriptive Application. Stanford, Ca.: Stanford University Press.

Langacker, R.W. (2001). What wH means. In A. Cienki, B.J. Luka \& M.B. Smith (eds.) Conceptual and Discourse Factors in Linguistic Structure. Stanford, Ca.: CSLI publications, 137-152.

Langacker, R.W. (2008). Cognitive Grammar: A Basic Introduction. Oxford / New York: Oxford University Press.

Langacker, R.W. (2011). Semantic motivation of the English auxiliary. In Panther, K.-U. \& G.

Radden (eds.) Motivation in Grammar and the Lexicon. Amsterdam / Philadelphia: John Benjamins, $29-47$.

Langacker, R.W. (2012). Substrate, system, and expression: aspects of the functional organization of English finite clauses. In Brdar, M., I. Raffaelli \& M.Z. Fuchs (eds.) Cognitive Linguistics between Universality and Variation. Newcastle upon Tyne (UK): Cambridge Scholars Publishing, 3-52.

Leonarduzzi, L. (2004). La subordonnée interrogative en anglais contemporain. Aix-en-Provence : Publications de l'Université de Provence.

Levinson, S.C. (2010). Questions and responses in Yélî Dnye, the Papuan language of Rossel Island. Journal of Pragmatics 42(10), 2615-2619. 
Méry, R. (1994). L'inversion sujet/auxiliaire en anglais sans antéposition d'un tiers élément. Sigma 16, 9-57.

Miller, P. (2000). Do auxiliaire en anglais : un morphème grammatical sans signification propre. In Carvalho, P. \& L. Labrune (eds.) Travaux linguistiques du Cerlico 13. Rennes: Presses Universitaires de Rennes, 119-147.

Minkova, D. (2004). Philology, linguistics, and the history of [hw] [w]. In Curzan, A. \& Emmons, K. (eds.) Studies in the History of the English Language II. Berlin / New York: Mouton de Gruyter, 7-46.

Nolan, F. (2006). Intonation. In Aarts, B. \& A. McMahon (eds.) The Handbook of English Linguistics, 433-457.

Ohala, J. J. (1984). An ethological perspective on common cross-language utilization of F0 of voice. Phonetica 41, 1-16.

Ohala, J.J. (1994). The frequency code underlies the sound symbolic use of voice pitch. In Hinton, L., J. Nichols \& J. J. Ohala (eds.) Sound symbolism. Cambridge: Cambridge UP, 325-347.

Palmer, F.R. (1987). The English Verb. 2nd edition. London \& New York: Longman.

Panther, K.-U. \& Thornburg, L. (1998). A cognitive approach to inferencing in conversation. Journal of Pragmatics 30, 755-769.

Panther, K.-U. \& Thornburg, L. (2007). Metonymy. In Geeraerts, D. \& H. Cuyckens (eds.) Oxford Handbook of Cognitive Linguistics. Oxford : Oxford University Press, 236-263.

Puckica, J. (2008). Sens et relations de sens dans les grammaires de construction. Lexis, E-Journal In English Lexicology, $\mathrm{n}^{\circ} 1$ : La polysémie / Polysemy.

URL : http://screcherche.univ-lyon3.fr/lexis/spip.php?article74

Puckica, J. (2015). L'argument de la pauvreté du stimulus linguistique et la construction des questions fermées en anglais. Faits de langues 45, 1, 91-118.

Quirk, R., Greenbaum, S., Leech, G., \& Svartvik, J. (1985). A Comprehensive Grammar of the English Language. London: Longman.

Searle, J.R. (1969). Speech Acts: An Essay in the Philosophy of Language. London / New York: Cambridge University Press.

Searle, J.R. (1979). Expression and Meaning: Studies in the Theory of Speech Acts. Cambridge: Cambridge University Press.

Stenström, A.-B. (1984). Questions and Responses in English Conversation. Lund Studies in English, vol. 68. Malmö : Liber Förlag.

Taylor, J.R. (2003). Linguistic Categorization. 3rd edition. Oxford, New York: Oxford University Press.

Ultan, R. (1969). Some general characteristics of interrogative systems. Working Papers in Language Universals I. MS. Stanford: Stanford University, 39-63a.

Vaissière, J. (1995). Phonetic explanations for cross-linguistic similarities. Phonetica 52, 123-130.

Välimaa-Blum, R. (2001). Intonation and interrogation in English: It's all a matter of defaults. In L'interrogation. Actes du colloque du 17/03/2001, Univ. Paris III. SESYLIA.

URL: https://alaesfrance.files.wordpress.com/2015/04/blum.pdf

Varela Pérez, J.R. (1997). The use of periphrastic do in Early Modern English negative declaratives: evidence from the Helsinki Corpus. Sederi VIII, 35-43. 
Wells, J.C. (2006). English Intonation: An Introduction. Cambridge: Cambridge University Press.

\section{NOTES}

1. Nous utilisons la numérotation des lignes du texte-corpus : (60) Did she ever talk about them? indique que cet exemple commence ligne 60.

2. On retrouve en partie ici les conditions de réussite ou de "félicité » (felicity conditions) de pragmaticiens comme J. Austin et J.R. Searle. Par ailleurs, il est à noter que certains actes de langage sont éminemment ritualisés et codifiés (comme ceux que peuvent exprimer marier, baptiser ou s'excuser) et que tous présentent plus ou moins des spécificités selon les langues et les cultures, de même que les règles de politesse varient selon les pays. Certains linguistes préfèrent ainsi parler de «script culturel » (Goddard \& Wierzbicka 2004).

3. Le terme assertion est quelque peu excessif et l'on pourrait préférer déclaration, comme en anglais, où l'on dit souvent statement. De même, si une phrase déclarative peut constituer un apport d'information de L1 à L2, c'est loin d'être toujours le cas. Comme le note Langacker (2008, 473) : «In and of itself, a statement carries with it nothing more than the baseline expectationcharacteristic of all expressions-of being heard and understood."

4. La question dite alternative, ex. Was she happy or (was she) unhappy?, peut être considérée comme un type mineur de question fermée. Elle ferme, elle aussi, le champ des réponses possibles par son contenu et ne constitue pas formellement un type distinct de la question polaire. La subordonnée correspondante est d'ailleurs introduite par whether (cf. 24-5), comme peut l'être la subordonnée interrogative polaire.

5. La notation catégorielle-fonctionnelle employée ici se veut surtout pratique : S(UBJ) pour sujet, COMP (complément), $\mathrm{AUX}_{\mathrm{F}}$ (auxiliaire fini), $\mathrm{V}_{\mathrm{NF}}$ (forme verbale non-finie), $\mathrm{V}_{\mathrm{INF}}$ (infinitif), $\mathrm{PC}_{\mathrm{S}}$ (Subject Predicative Complement, i.e. attribut du sujet), etc.

6. Les données mentionnées par Quirk et al. $(1985,807)$ pour l'anglais britannique et celles de Hirschberg $(2000)$ mentionnées par Välimaa-Blum $(2001,10)$ pour l'anglais américain suggèrent qu'au moins env. $40 \%$ des questions fermées auraient une intonation descendante.

7. A la portée large (globale) de la question, qui s'étend à toute la proposition, peut s'ajouter une portée plus restreinte (locale). À la question Will you call David tomorrow?, on peut ainsi répondre No, I'll call him next week ( = 'not tomorrow'), mais aussi No, I'll call Peter ( = 'not David').

8. En termes logiques, la question porte sur la «valeur de vérité » du contenu propositionnel. Le terme réalité peut sembler préférable à existence et plus encore à vérité, qui deviennent embarrassants lorsque la question n'est pas une demande d'information, mais plutôt une offre (Shall we go?), une requête (Could you please stay?) ou un conseil (Would you advise me to stay?). D'où la distinction parfois proposée entre les «information questions » et les «direction questions » (ex. Huddleston $2002: 876-8$ ), mais cette distinction est assez artificielle - elle suggère par ex. qu'une demande de conseil n'est pas une demande d'information - et ne règle rien.

9. Cf. Wells (2006: 42-3) : "[...] a wh question can also be said with a non-fall : a rise or, less commonly, a fall-rise. This has the effect of making it more gentle, kindly, encouraging, sympathetic or deferential, as opposed to the businesslike fall. We call this tone meaning the encouraging rise ». Quirk et al. (1985: 817) notent que sur un ensemble de 858 questions en wh dans un corpus d'anglais britannique oral, 775 avaient une intonation descendante, soit plus de $90 \%$. L'intonation des questions ouvertes semblerait donc moins variable que celle des questions fermées. Ceci pourrait être lié au fait que le ton descendant est globalement le ton par défaut.

10. Cette «mise en avant " demanderait à être davantage étudiée : certains grammairiens y voient une forme de focalisation, tout particulièrement dans le cas des mots en wh (ex. Ultan 
1969), mais d'autres parlent «au contraire » de topicalisation (ex. Langacker 1991:506) ou de thématisation (cf. P. Cotte, ce volume).

11. Rappelons que la graphie wh- est une modification moyen-anglaise des formes en $h w$ - du vieil-anglais, ex. hwcet (what), hwa (who), hwi (why), hwilc (which), hwcer, hwar (where), etc., avec, à nouveau, le cas particulier de how, v.-angl. $h u$, dont on reconstruit *hwo sur la base d'une comparaison avec d'autres langues germaniques (vx.-sax. hwo, got. hvaiwa, vx.-haut-alld. (h)weo, (h)wio, alld. wie). Ajoutons que la graphie $h w$ - permet de mieux voir le lien de ces formes avec celles en qu- des langues romanes, auxquelles elles sont liées par la (première) loi de Grimm : p.i.e. $* / \mathrm{kw} />$ p.-germ. $* / \mathrm{xw} />$ v.-angl. /hw/. Sur l'histoire de wh-, voir notamment Minkova (2004). 12. Il existe aussi des contre-exemples. Par exemple, on dit to come up with an answer, ce que relèvent Lakoff \& Johnson (id., 137) sans en donner une explication très satisfaisante. En français, on soulève des questions ou problèmes, mais on peut aussi les poser.

13. Chen (2013) propose une autre hypothèse pour rendre compte des divers cas d'inversion sujet-auxiliaire, suivant laquelle il existerait deux constructions sémantiquement distinctes : (a) une construction sans élément antéposé, nommée "Auxiliary Subject Construction ", qui marquerait les «modes non indicatifs »; et (b) une construction avec un élément antéposé (ex. mot en wh- ou so), nommée «X Auxiliary Subject Construction ", qui permettrait quant à elle une focalisation sur l'élément mis en position initiale. C'est une proposition pour le moins discutable : selon Chen (2013: 4), par exemple, une question fermée (ex. Do you like coffee?) ne peut " clairement » pas être au mode indicatif, mais seulement au « mode interrogatif ».

14. La grammaticalisation de $d o_{\mathrm{AUX}}$ a été longue et tardive. On trouve des traces écrites de l'emploi de $d o_{A U X}$ dès le $13^{\mathrm{e}}$ siècle dans les dialectes du sud-ouest de l'Angleterre (cf. Filppula \& Klemola 2007: 220), mais ce n'est qu'à partir du $16^{\mathrm{e}}$ siècle que l'emploi de $\mathrm{do}_{\mathrm{AUX}}$ semble véritablement se répandre. Au début du $17^{\mathrm{e}}$ siècle, dans les œuvres de Shakespeare (1564-1616) ou Jonson (1572-1637), les verbes lexicaux peuvent encore être directement construits avec not ou placés devant le sujet dans des questions. En 1700, do n'est encore employé que dans $55 \%$ des propositions négatives sans autre auxiliaire dans le Helsinki Corpus selon Varela Pérez (1997). Il semble donc qu'il faille attendre le $18^{\mathrm{e}}$ siècle pour voir l'emploi de do devenir obligatoire (nonmarqué) dans la langue standard en l'absence de tout autre auxiliaire. Voir aussi la contribution de $\mathrm{S}$. Hancil à ce volume.

15. A l'origine des approches constructionnelles modernes de la grammaire, il y a notamment les travaux de Ch. Fillmore, P. Kay et collaborateurs sur le problème des structures - en particulier, les innombrables locutions (idioms) - qui suivent certains principes grammaticaux mais contreviennent à d'autres (cf. Croft \& Cruse 2004, chap.9).

16. Concernant les polarités respectives de la proposition hôte et du QT, sur un total de 754 QT tirés d'un corpus d'anglais britannique (oral et écrit), Kim \& Ann (2008) trouvent la répartition suivante : POS-NEG 467 (c. 62 \%), NEG-POS 196 (c. $26 \%$ ), POS-POS 88 (c. $11.5 \%$ ), NEG-NEG 3 (c. 0,5\%). Les QT de polarité inversée dominent largement : $88 \%$ du total, contre $12 \%$ pour ceux de polarité constante, qui sont presque exclusivement des QT positifs adjoints à des propositions positives. Généralement, ces derniers ont une intonation (légèrement) montante et expriment un reproche ou sarcasme (cf. Quirk et al. 1985, 812). Ils se différencient donc également sur ce point des QT de polarité inversée.

17. Parfois, l'assurance du L1 peut s'interpréter comme une marque de défiance, voire de menace (domination) : ex. We're not so Diffferent, ÀRE we? ('tu ne peux pas dire le contraire', 'tu ne vas pas oser me dire le contraire').

18. Selon Levinson (2010), au moins $50 \%$ des questions polaires dans les corpus d'anglais oral seraient de forme déclarative. L'auteur cite une étude de Stenström (1984) selon laquelle $75 \%$ des questions polaires de forme déclarative auraient une intonation descendante en anglais. Cette étude, cependant, ne paraît pas distinguer les questions déclaratives des questions indirectes. 
19. Cette hypothèse n'est pas sans rappeler la proposition de Searle (1969: 68), pour qui il est possible d'accomplir un acte de langage sans utiliser de marqueur de cet acte si l'énoncé et le contexte indiquent clairement que la «condition essentielle » de cet acte est satisfaite. Or, la condition essentielle de la question, pour Searle (id., 66), est que L1 tente d'obtenir une information de L2.

20. La distribution de whether, si ce n'est pour le fait que ce mot en wh ne s'emploie plus que dans des subordonnées en anglais moderne, peut sembler plus proche de why que de if. Une subordonnée interrogative en if est nécessairement finie et elle ne peut être ni sujet (sauf extraposition), ni attribut, ni complément prépositionnel, ni apposée. Une subordonnée en whether ou why peut être finie ou non-finie et peut occuper toutes ces fonctions. L'opposition interrogative fermée $v$. ouverte est, en tout état de cause, une simplification.

21. Le remplacement de la subordonnée par someone n'est possible que si l'on considère know dans le sens de 'connaître (qqch / qqn)' plutôt que de 'savoir (qqch / *qqn)', soit dans un sens qui n'est pas celui de (c).

\section{RÉSUMÉS}

Le présent article traite des questions en anglais suivant une approche qui s'inscrit dans le cadre de la linguistique cognitive. Les principaux types de question y sont discutés, ainsi que les diverses structures au moyen desquelles elles sont exprimées: propositions interrogatives indépendantes fermées et ouvertes, question tags, questions déclaratives, questions-répliques (echo questions) et questions indirectes. L'intonation des questions, l'inversion sujet-auxiliaire et le rôle de l'auxiliaire do font partie des points abordés. La distinction, parfois délicate, entre les propositions subordonnées interrogatives et les constructions relatives dites libres est également discutée. Les diverses structures analysées sont envisagées comme des instances de constructions symboliques, dotées à la fois de propriétés formelles et sémantiques-pragmatiques. Considérée sous cet angle, la grammaire n'est pas indépendante du sens mais apparaît, tout au contraire, fondamentalement signifiante et motivée.

This article analyses questions in English from a Cognitive Linguistics perspective. The main kinds of question are discussed as well as the various structures used to express them: main interrogative clauses, both closed and open, tag questions, declarative questions, echo questions, and indirect questions. The intonation of questions, subject-auxiliary inversion, and the role of the auxiliary do are among the topics discussed. The sometimes subtle distinction between subordinate interrogative clauses and free relative constructions is also touched upon. The various structures which are dealt with are considered as instances of symbolic constructions with their own formal and semantic-pragmatic properties. From that perspective, grammar is not independent of meaning but instead appears to be fundamentally meaningful.

\section{INDEX}

Keywords : questions, interrogative sentences, grammatical constructions, cognitive grammar Mots-clés : questions, phrases interrogatives, constructions grammaticales, grammaire cognitive 
AUTEUR

JÉRÔME PUCKICA

LIDILEM, Université Grenoble Alpes

jerome.puckica@univ-grenoble-alpes.fr 